\title{
Two-dimensional $\mathrm{WS}_{2}$ Crystals at Predetermined Locations by Anisotropic Growth During Atomic Layer Deposition
}

\author{
Benjamin Groven, ${ }^{\mathrm{a}}$ Yoann Tomczak, ${ }^{\mathrm{a}, \uparrow}$ Marc Heyns, ${ }^{\mathrm{a}, \mathrm{b}}$ Iuliana Radu, ${ }^{\mathrm{a}}$ and Annelies Delabie*a,c \\ a. imec, B-3001 Leuven, Belgium. \\ b. Department of Materials Engineering, KU Leuven (University of Leuven), B-3001 Leuven, \\ Belgium. \\ c. Department of Chemistry, KU Leuven (University of Leuven), B-3001 Leuven, Belgium \\ † Current address: ASM Belgium, B-3001 Leuven, Belgium. \\ *Corresponding author: Prof. dr. Annelies Delabie (annelies.delabie@imec.be)
}

\begin{abstract}
Anisotropic growth of two-dimensional (2D) tungsten disulfide $\left(\mathrm{WS}_{2}\right)$ crystals occurs during atomic layer deposition (ALD) from $\mathrm{WS}_{2}$ seeds at predetermined locations on large area dielectric substrates. The number of ALD reaction cycles determines the lateral dimensions of the $\mathrm{WS}_{2}$ crystals. This 2D synthesis approach is compatible with temperature sensitive structures due to the low deposition temperature, and can be extended to other 2D materials and heterostructures thereof. The crystallinity of the seed and the selectivity of ALD precursors toward seeds and underlying starting surface affect the structural quality of the 2D crystals.
\end{abstract}

\section{Introduction}

Two-dimensional (2D) materials, with graphene and the transition metal dichalcogenides as the most studied representatives, represent a class of materials with layered structures ${ }^{1}$. A wide variety of $2 \mathrm{D}$ materials exists with versatile properties, that moreover can be tuneable through the composition and number of layers. $2 \mathrm{D}$ materials are under investigation for numerous applications, including nano-electronics, optoelectronics, energy storage, and catalysis ${ }^{2}$. The semiconducting 2D materials like tungsten- and molybdenum disulfide $\left(\mathrm{WS}_{2}\right.$ and $\left.\mathrm{MoS}_{2}\right)$ are of great interest for application in nanoelectronic devices as complement to silicon, in view of their monolayer thickness, large band gap values, low dielectric constants, structural stability and self-passivating nature of the basal plane ${ }^{2,3}$. The implementation of 2D semiconductors in nanoelectronic devices requires industrially relevant deposition techniques to synthesize these materials on large area structures. Obtaining high structural quality becomes important, as impurities, defects and grain boundaries can affect or degrade the carrier mobility in the 2D semiconductor. In addition, monolayer thickness control is required as the properties can depend on the number of monolayers.

Today, most studies of 2D semiconductor deposition focus on top-down approaches using chemical vapor deposition (CVD) at moderate to high deposition temperatures $\left(550^{\circ} \mathrm{C}-1150^{\circ} \mathrm{C}\right)^{4}$. However, direct growth on amorphous substrates yields polycrystalline structures, and randomly located grain boundaries can intersect the functional area of electronic devices and hamper the mobility of carriers through scattering5. Monocrystalline layers can be deposited by epitaxy, but this requires a template for epitaxial seeding 6 . Also, in an additional process step the 2D material needs to be transferred from template to target substrate. Developing industry compatible transfer processes that preserve the 2D material properties, is currently not straightforward 7 .

In an alternative approach, single 2D semiconductor crystals are created from the bottom up, at predetermined locations and only where needed in the final device. This way, grain boundaries are in principle kept outside the active area (Figure 1). This socalled controlled seeding approach exploits the anisotropic reactivity of $2 \mathrm{D}$ crystals, and relies on selective lateral growth of $2 \mathrm{D}$ crystals starting from a catalyst or reactive seed 
that is present at predetermined locations. Anisotropic growth can occur when crystalline materials grow along a preferential crystallographic direction, and has been used to control the shape and/or texture during CVD ${ }^{8-11}$, and epitaxial growth ${ }^{12,13}$. While this approach can in principle ensure the monocrystalline semiconductor structure in the device area, it can at the same time simplify the integration process flow by eliminating subsequent patterning and etch processes, as such reducing the risk of defect formation in the 2D material during patterning ${ }^{14}$.

Recent reports demonstrate the controlled seeding of $\mathrm{MoS}_{2}, \mathrm{WS}_{2}$ and tin disulfide $\left(\mathrm{SnS}_{2}\right)$ crystals ${ }^{15-21}$, with state-of-the-art electronic properties ${ }^{22}$. However, these approaches either require high temperatures as they rely on chemical vapor transport (CVT) or CVD $\left(\geq 650^{\circ} \mathrm{C}\right.$ ) or employ metal catalysts like Platinum. This is not compatible with direct implementation of controlled seeding in devices in back end of line (BEOL) structures. Atomic Layer Deposition (ALD) at seeded growth sites could provide an attractive solution (Figure 1). ALD is an industrially relevant deposition technique that can operate at low deposition temperatures. It can provide atomic scale precision and selectivity as it relies on self-limiting surface reactions ${ }^{23}$. However, while deposition of 2D materials by ALD is under extensive research, the ALD of 2D materials from seeds has not yet been reported in literature 24,25 . Moreover, anisotropic growth by ALD has been reported only for a very limited number of processes $9,26,27$.

This article explores the anisotropic growth of $\mathrm{WS}_{2}$ crystals during ALD from $\mathrm{WS}_{2}$ seeds on a dielectric starting surface. The concept is illustrated in figure 1. According to the proposed concept, the lateral dimensions of the $\mathrm{WS}_{2}$ crystals increase with the number of ALD reaction cycles, while the height of the $\mathrm{WS}_{2}$ crystals is mainly determined by the initial seed and remains nearly constant during ALD (Figure 1). In this paper, a proof of concept for controlled seeding of $\mathrm{WS}_{2}$ crystals during ALD is demonstrated, making use of a complementary metal-oxide-semiconductor (CMOS) compatible seed and a deposition temperature of $450{ }^{\circ} \mathrm{C}$ close to BEOL processing restriction of $400{ }^{\circ} \mathrm{C}$.

Si nanodots

by patterning Si layer
$\mathrm{WS}_{2}$ seeds

by selective conversion
Anisotropic WS $\mathrm{W}_{2}$ growth during PEALD

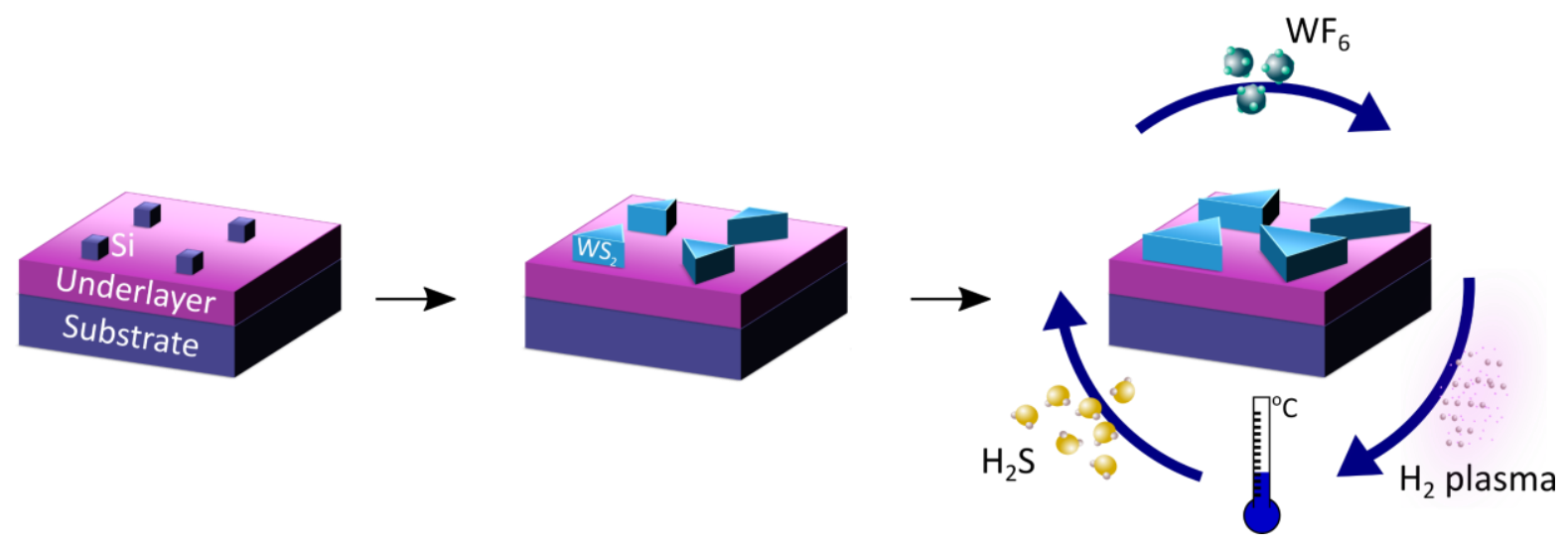

Figure 1. Schematic of the proposed controlled seeding concept: (i) Patterning of blanket Si layer to form arrays of $\mathrm{Si}$ nanodots at predetermined locations on $300 \mathrm{~mm}$ substrates. (ii) The Si nanodots are being consumed to form monocrystalline $\mathrm{MX}_{2}$ seeds (e.g., $\mathrm{WS}_{2}$ ) at well-defined locations by selective conversion process. (iii) Selective lateral growth from predetermined $\mathrm{MX}_{2}$ seeds by industrial deposition technique such as CVD or ALD (e.g., WS 2 PEALD from $\mathrm{WF}_{6}, \mathrm{H}_{2}$ plasma and $\mathrm{H}_{2} \mathrm{~S}$ ). 


\section{Experimental set-up}

Both the selective conversion of Si nanodot to $\mathrm{WS}_{2}$ seed and the $\mathrm{WS}_{2}$ PEALD take place inside a hot-wall, showerhead-type ASM PECVD reactor with direct (RF) plasma capability, connected to an Eagle12 $2^{\mathrm{TM}}$ platform. As such, air exposure between the seed formation and PEALD is avoided. Both processes occur at a susceptor temperature of $450^{\circ} \mathrm{C}$, whereas wall and showerhead temperature remain constant at $185^{\circ} \mathrm{C}$ and $225^{\circ} \mathrm{C}$, respectively.

The $\mathrm{WS}_{2}$ seeds are created by selective conversion of a prepatterned blanket Si layer on a dielectric substrate, to form metallic $\mathrm{W}$ by the reaction of the $\mathrm{Si}$ nanodots with $\mathrm{WF}_{6}$ at $450^{\circ} \mathrm{C}$, followed by in-situ sulfidation by $\mathrm{H}_{2} \mathrm{~S}$ at $450^{\circ} \mathrm{C}^{28}$. The conversion process from $\mathrm{Si}$ layers yields polycrystalline $W_{2}$ films with grain size of 5-10 nm. Here, the same conversion process from Si nanodots is investigated, with the aim to examine if the formation of nanoscale monocrystalline $\mathrm{WS}_{2}$ seeds is possible. Amorphous Si is deposited by molecular beam epitaxy (MBE) on $300 \mathrm{~mm} \mathrm{Si(100)} \mathrm{substrates} \mathrm{coated} \mathrm{with} 10 \mathrm{~nm}$ dielectric layer of either aluminium oxide $\left(\mathrm{Al}_{2} \mathrm{O}_{3}\right)$ or silicon nitride $\left(\mathrm{Si}_{3} \mathrm{~N}_{4}\right)$. The patterning process to obtain arrays of Si nanodots relies on immersion lithography and fluorocarbon $\left(\mathrm{CF}_{\mathrm{x}}\right)$-based plasma-assisted etch chemistry ${ }^{29}$. Next, the surface of the Si nanodots is oxidized in an ozone and water $\left(\mathrm{O}_{3}: \mathrm{H}_{2} \mathrm{O}\right)$ solution and passivated with hydrogen by exposure to a $0.5 \%$ aqueous hydrogen fluoride (HF) solution ${ }^{29}$. Native oxide removal proves essential to enable subsequent reaction $\mathrm{WF}_{6}$ with $\mathrm{Si}$, as shown in earlier work ${ }^{28}$.

Since the transition atom $\mathrm{W}$ requires a partial reduction from the $+\mathrm{VI}$ oxidation state in $\mathrm{WF}_{6}$ to $+\mathrm{IV}$ in $\mathrm{WS}_{2}$, the Si nanodots act as the reducing agent for the $\mathrm{W}$ atom during the conversion mechanism, according to reaction pathways $(1,2)$. Both reactions are likely to proceed at the deposition temperature used here (i.e., $450^{\circ} \mathrm{C}$ ), as reported in literature ${ }^{30}$.

$$
\begin{array}{ll}
2 \mathrm{WF}_{6(\mathrm{~g})}+3 \mathrm{Si}_{(\mathrm{s})} & \rightarrow 2 \mathrm{~W}_{(\mathrm{s})}+3 \mathrm{SiF}_{4(\mathrm{~g})} \\
\mathrm{WF}_{6(\mathrm{~g})}+3 \mathrm{Si}_{(\mathrm{s})} & \rightarrow \mathrm{W}_{(\mathrm{s})}+3 \mathrm{SiF}_{2(\mathrm{~g})}
\end{array}
$$

The conversion process is driven by the diffusion of $\mathrm{F}$ through the formed metallic $\mathrm{W}$, and the diffusion of $\mathrm{SiF}_{\mathrm{x}}$ in the opposite direction, towards the surface. For nanoscale $\mathrm{Si}$ structures considered here, diffusion is not a limiting factor. The Si nanodots are being consumed completely and metallic $\mathrm{W}$ nanodots form by a self-limiting conversion process. The Si areal density determines the amount of deposited W: as soon as all Si is consumed, the deposition of $\mathrm{W}$ becomes self-terminating. Hence, the dimensions of the $\mathrm{W}$ nanodots can be controlled by the Si nanodot dimensions.

During the $\mathrm{H}_{2} \mathrm{~S}$ reaction, the W nanodots oxidize to $\mathrm{WS}_{2}$ seeds by reaction (3). Both Rutherford backscattering spectroscopy (RBS) and X-ray photoelectron spectroscopy (XPS) confirm that the majority of $\mathrm{W}$ atoms reside in the +IV oxidation state when $\mathrm{WS}_{2}$ is grown from a $S i$ sacrificial layer, with a $S / W$ ratio of $(1.8 \pm 0.2)(X P S)^{31}$.

$$
\mathrm{W}_{(\mathrm{s})}+2 \mathrm{H}_{2} \mathrm{~S}_{(\mathrm{g})} \quad \rightarrow \mathrm{WS}_{2(\mathrm{~s})}+2 \mathrm{H}_{2(\mathrm{~g})}
$$

The Si to $\mathrm{WS}_{2}$ conversion process displays high selectivity, as $\mathrm{WS}_{2}$ crystals form only where the Si nanodot is present and reaction with the dielectric layer is inhibited. Indeed, the amount of deposited $\mathrm{W}$ on $\mathrm{Si}_{3} \mathrm{~N}_{4}$ after $\mathrm{WF}_{6}$ exposure in absence of Si precursor remains below the detection limit of RBS (i.e., $<10^{13} \mathrm{~W}$ atoms $/ \mathrm{cm}^{2}$ ).

Afterwards, the $\mathrm{WS}_{2}$ seeds are exposed to plasma enhanced (PE)ALD ternary reaction cycle of $\mathrm{WF}_{6}$ (CAS no. 7783-82-6), $\mathrm{H}_{2}$ plasma (CAS no. 1333-74-0) and $\mathrm{H}_{2} \mathrm{~S}(99.9 \%$ pure $\mathrm{H} 2 \mathrm{~S}, 10 \%$ in He, CAS no. 7783-06-4 and 7440-59-7). The PEALD reaction cycle consists of $10 \mathrm{~s}$ of $\mathrm{WF}_{6}$ exposure $(50 \mathrm{sccm}), 10 \mathrm{~s}$ of $\mathrm{H}_{2}$ plasma discharge $(500 \mathrm{sccm})$ at $100 \mathrm{~W}$, and 60 $\mathrm{s}$ of $\mathrm{H}_{2} \mathrm{~S}$ exposure (in $\mathrm{He}, 695 \mathrm{sccm}$ ) at a constant reactor chamber pressure of 2 Torr and a susceptor temperature of $450^{\circ} \mathrm{C}$. Each of the precursor and reactant species flow into the reactor chamber sequentially. The $\mathrm{WS}_{2}$ film grows through self-limiting surface reactions, as verified in earlier work ${ }^{32}$. Prior to unloading the wafers, they cool down in $\mathrm{N}_{2}$-purged load-lock for 15 minutes.

The $\mathrm{WS}_{2}$ PEALD process displays a bulk GPC of $(3.3 \pm 0.4) \times 10^{13} \mathrm{~W}$ atoms $/ \mathrm{cm}^{2} / \mathrm{cycle}$ at $450^{\circ} \mathrm{C}$ (i.e., the equivalent of $0.03 \mathrm{ML} /$ cycle). A careful control over the $\mathrm{H}_{2}$ plasma and $\mathrm{H}_{2} \mathrm{~S}$ reactions, results in a well-controlled composition (i.e., S/W ratio of $1.9 \pm 0.1$ ) of the deposited layers. The $\mathrm{H}_{2}$ plasma reaction enables the reduction of $-\mathrm{W}^{6+} \mathrm{F}_{\mathrm{x}}$ surface species, 
but needs to be mild ( $100 \mathrm{~W}, 10 \mathrm{~s})$ to minimize sub-surface reduction of the $\mathrm{WS}_{2}$ layers, as detailed in earlier work ${ }^{32,33}$.

RBS and XPS investigate the composition of the crystals. RBS uses a $1.52 \mathrm{MeV} \mathrm{He}^{+}$ion beam at $25 \mathrm{nA}$ beam current to quantify the areal density of W and S. The incident ion beam impinges at $11^{\circ}$ with respect to the sample normal with detector placed at a scattering angle of $170^{\circ} 34$. XPS measurements utilize a Theta300 system from Thermo Instruments with monochromatized $\mathrm{Al} \mathrm{K \alpha} \mathrm{X}$-ray source $(1486.6 \mathrm{eV})$ and spot size of 400 $\mu \mathrm{m}$ in angle-resolved (AR) mode. After referencing the binding energies to the C1s peak and correcting for Shirley background, all recorded spectra are fitted using pseudo-Voight functions.

Atomic force microscopy (AFM) and scanning electron microscopy (SEM) measure the lateral and vertical dimensions of the $\mathrm{WS}_{2}$ crystals. AFM uses a Nanoscope $\mathrm{V}$ instrument from ICON PT (Bruker) in tapping mode equipped with $7 \mathrm{~nm}$ radius tip (Olympus OCMLAC160TS). The lateral dimension of the $\mathrm{WS}_{2}$ crystals is approximated by the diameter of the circle with equivalent surface area. The height of the $\mathrm{WS}_{2}$ crystals is inferred from the AFM height profiles. For SEM, secondary electron micrographs are collected using FEI Helios 1200 operating at beam energies and currents up to $10 \mathrm{kV}$ and $0.2 \mathrm{nA}$, respectively.

The crystallinity and structure of the $\mathrm{WS}_{2}$ crystals is investigated by Raman spectroscopy using a Horiba Jobin-Yvon HR800 Raman instrument at $532 \mathrm{~nm}$ laser excitation wavelength. To evaluate the $2 \mathrm{D}$ structure of the $\mathrm{WS}_{2}$, the Raman laser operates at $\sim 2 \mathrm{~mW}$ laser power with a $1 \mu \mathrm{m}^{2}$ laser spot size. The Raman spectra are acquired for $200 \mathrm{~s}$ using a $300 \mu \mathrm{m}$ confocal hole with a $1800 \mathrm{gr} / \mathrm{mm}$ grating. After normalizing the recorded spectra to the Si peak $\left(300 \mathrm{~cm}^{-1}\right)$ and applying background correction through a blanket Si reference measurement, all peaks are deconvoluted into the individual Raman modes by using Voight functions.

\section{Results and discussion}

First, AFM reveals the structure of the Si nanodots and $\mathrm{WS}_{2}$ seeds. While a range of arrays of Si nanodots with different dimensions are present, the focus lies on two arrays with the smallest structures, indicated as Array 1 and Array 2 (Table 1). After lithography, etch and photoresist and bottom anti-reflective coating strip, the Si nanodots covered with native $\mathrm{SiO}_{2}$ are $1.7 \pm 0.3 \mathrm{~nm}$ high and $12 \pm 6 \mathrm{~nm}$ in diameter for Array 1, with a pitch of $300 \mathrm{~nm}$ (AFM, Table 1). In Array 2, the Si nanodots are $5.9 \pm 0.1 \mathrm{~nm}$ high and $48 \pm 1 \mathrm{~nm}$ in diameter with a pitch of $300 \mathrm{~nm}$ (AFM, Table 1, and Figure 2a, Figure S2 in SI).

Table 1. Average lateral size (i.e., diameter) and height of prepatterned Si nanodots and $\mathrm{WS}_{2}$ seeds in Array 1 and 2 after selective conversion and PEALD.

\begin{tabular}{|c|c|c|c|c|c|c|}
\hline & & Array 1 & \multirow[b]{2}{*}{ 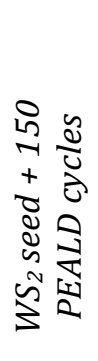 } & \multirow[b]{2}{*}{ 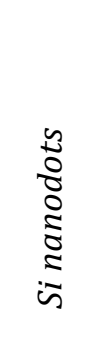 } & \multirow[b]{2}{*}{ 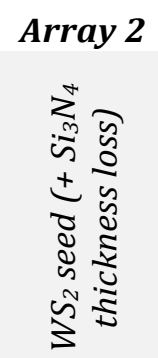 } & \multirow[b]{2}{*}{ 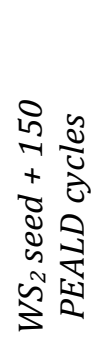 } \\
\hline & 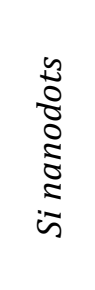 & 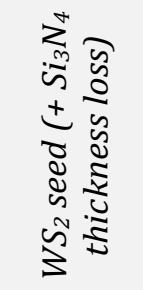 & & & & \\
\hline $\begin{array}{l}\text { Average } \\
\text { lateral size } \\
(\mathrm{nm})\end{array}$ & $\begin{array}{l}12 \\
\pm 6\end{array}$ & $\begin{array}{l}38 \\
\pm 4\end{array}$ & $\begin{array}{l}91 \\
\pm 7\end{array}$ & $\begin{array}{l}48 \\
\pm 1\end{array}$ & $\begin{array}{l}59 \\
\pm 1\end{array}$ & $\begin{array}{l}119 \\
\pm 11\end{array}$ \\
\hline $\begin{array}{l}\text { Average } \\
\text { height } \\
\text { (nm) }\end{array}$ & $\begin{array}{c}1.7 \\
\pm 0.3\end{array}$ & $\begin{array}{c}9 \\
\pm 1\end{array}$ & $\begin{array}{l}20 \\
\pm 8\end{array}$ & $\begin{array}{c}5.9 \\
\pm 0.1\end{array}$ & $\begin{array}{l}12.0 \\
\pm 0.4\end{array}$ & $\begin{array}{l}19 \\
\pm 8\end{array}$ \\
\hline
\end{tabular}



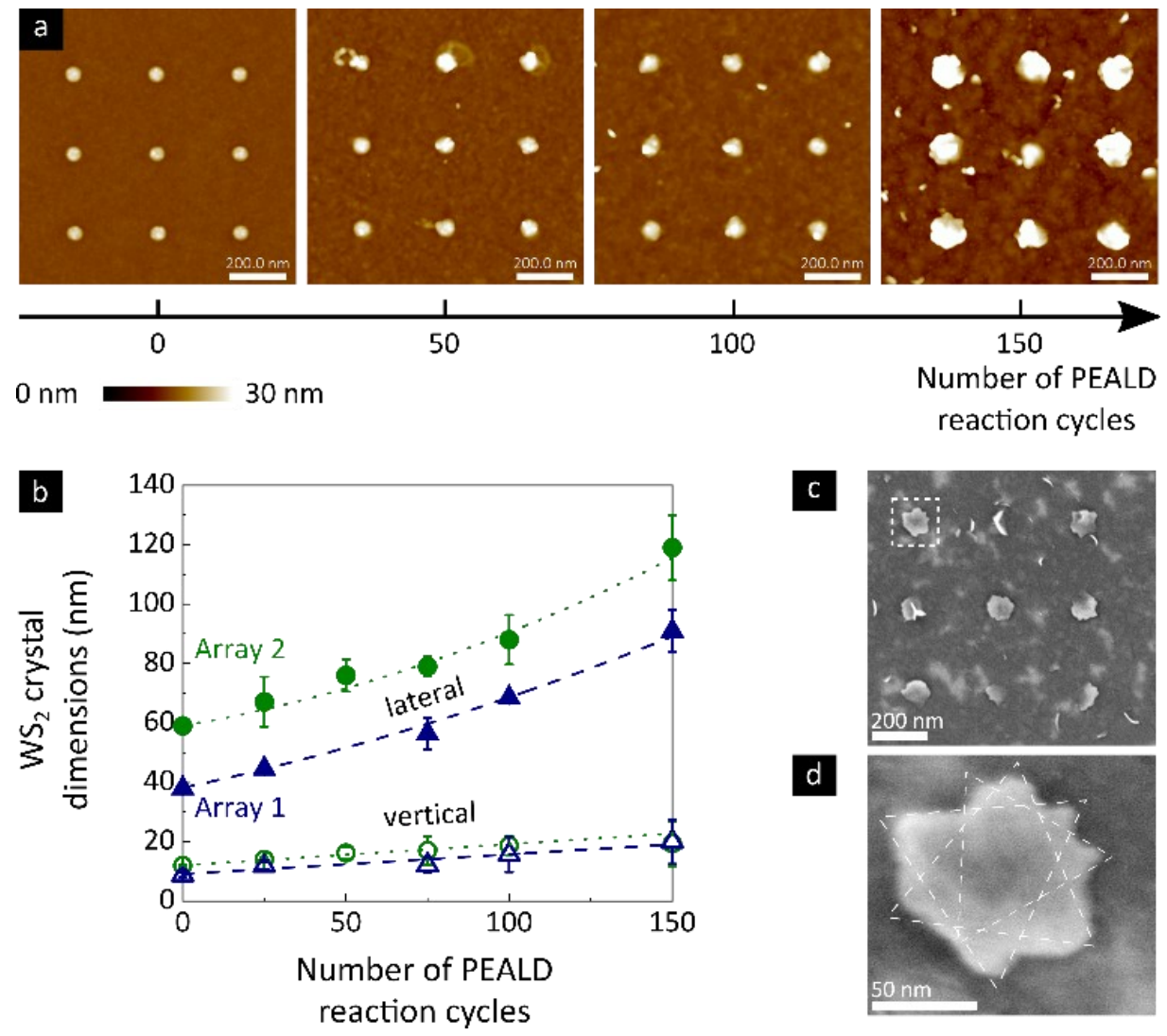

Figure 2. (a) Atomic force micrographs $(1 \mu \mathrm{m} \times 1 \mu \mathrm{m})$ of $\mathrm{WS}_{2}$ crystal array for increasing number of PEALD reaction cycles (i.e., 0, 50, 100, and 150) and fixed WS 2 seed starting dimension of $59 \pm 1$ $\mathrm{nm}$. Lateral and vertical scale bars are set to $200 \mathrm{~nm}$ and $30 \mathrm{~nm}$, respectively. (b) Corresponding lateral and vertical dimensions of $\mathrm{WS}_{2}$ crystals in closed and open symbols for two starting $\mathrm{WS}_{2}$ seed diameters of $38 \pm 4 \mathrm{~nm}$ (triangles) and $59 \pm 1 \mathrm{~nm}$ (circles), as a function of the number of PEALD reaction cycles. Dotted lines guide the eye, with quadratic and linear regression for lateral and vertical dimensions; (c-d) For 150 PEALD reaction cycles, the majority of $\mathrm{WS}_{2}$ seeds develop (0002) texture, as determined from scanning micrographs. Dotted lines mark the WS $\mathrm{S}_{2}$ layers within a crystal with different in-plane orientation along [0001] crystal direction. All depositions occur at $450{ }^{\circ} \mathrm{C}$ on $10 \mathrm{~nm} \mathrm{PEALD} \mathrm{Si}_{3} \mathrm{~N}_{4}$.

After $\mathrm{HF}$ exposure removing the native $\mathrm{SiO}_{2}$ and selective conversion process into $\mathrm{WS}_{2}$, the height and diameter of the originating Si nanodots define the $\mathrm{WS}_{2}$ seed dimensions. A volume expansion occurs according to the reaction stoichiometry and due to the difference in density for $\mathrm{Si}$ and $\mathrm{WS}_{2}$. The conversion of the Si nanodots into $\mathrm{WS}_{2}$ occurs through reaction pathways (1-3). The Si from the Si nanodots is fully consumed after the selective conversion process (XPS, not shown), in line with the results for Si layers ${ }^{29}$.

The volume expansion of the structures during the conversion process agrees with previous studies of the selective conversion process ${ }^{31}$. For a $1.7 \mathrm{~nm}$ high Si nanodot, the expected height for the $W_{2}$ seeds is $\sim 3.2 \mathrm{~nm}$, corresponding to five $\mathrm{WS}_{2}$ monolayers according to a monolayer thickness of $0.62 \mathrm{~nm}^{31}$. However, after the HF exposure and $\mathrm{WS}_{2}$ conversion process, the height of the structures increases to $9 \pm 1 \mathrm{~nm}$ in Array 1 and $12.0 \pm 0.4 \mathrm{~nm}$ in Array $2(\mathrm{AFM}$, Table 1 and Figure S2 in SI). Note that the height as measured by AFM not only includes the $\mathrm{WS}_{2}$ seed height but also a few nm of $\mathrm{Si}_{3} \mathrm{~N}_{4}$ thickness loss due to $\mathrm{Si}_{3} \mathrm{~N}_{4}$ overetching during the HF exposure. As the $\mathrm{Si}_{3} \mathrm{~N}_{4}$ layer is partly oxidized due to air exposure, there is limited HF etch selectivity between the $\mathrm{Si}_{3} \mathrm{~N}_{4}$ underlayer and the native $\mathrm{SiO}_{2}$ layer present on the $\mathrm{Si}$ nanodots. The width of the structures also increases during the conversion process, up to a value of $38 \pm 4 \mathrm{~nm}$ for Array 1 and $59 \pm 1 \mathrm{~nm}$ for Array 2 (Table 1). Note that the width of the smallest structures is difficult to measure accurately by AFM. 
The $\mathrm{WS}_{2}$ seeds are prone to oxidation during air exposure. The $\mathrm{S} / \mathrm{W}$ ratio of the seeds after air exposure is only $0.5 \pm 0.1$ (XPS, Figure $3 a-b$ ). $W_{2}$ layers fabricated by the same conversion process from blanket, non-patterned Si layers have been shown to be more stable during air exposure due to lower surface to bulk ratio, as the $\mathrm{S} / \mathrm{W}$ ratio is $1.8 \pm 0.2$ after air exposure ${ }^{31}$. Therefore, one can assume that smaller structures formed here also consist of $\mathrm{WS}_{2}$ before air exposure. Hence, to avoid issues with oxidation of $\mathrm{WS}_{2}$ seeds during anisotropic growth by PEALD, the PEALD process occurs in the same reactor as the conversion process hereby avoiding air exposure. Note that the characterization of the crystallinity (mono or polycrystalline) of the $\mathrm{WS}_{2}$ seeds is extremely difficult at this point due to their small size.

Second, anisotropic growth from the $\mathrm{WS}_{2}$ seeds occurs during the subsequent $\mathrm{WS}_{2}$ PEALD process (Table 1 and Figure 2). This lateral growth process proceeds uniformly over Array 1 and 2. After $150 \mathrm{WS}_{2}$ PEALD reaction cycles, the lateral size of the $\mathrm{WS}_{2}$ crystals increases from $59 \pm 1 \mathrm{~nm}$ to $119 \pm 11 \mathrm{~nm}$ in Array 2 (Figure $2 \mathrm{~b}$ ). At the same time, the height of the structures increases from $12.0 \pm 0.4$ to $19 \pm 8 \mathrm{~nm}$ (incl. height of $\mathrm{WS}_{2}$ crystal and $\mathrm{Si}_{3} \mathrm{~N}_{4}$ recess, Figure $2 \mathrm{~b}$ ).

The $\mathrm{WF}_{6}$ adsorption on the surface is followed by surface reactions that occur primarily at the edges of the growing $2 \mathrm{D} \mathrm{WS}_{2}$ crystals ${ }^{33,35}$. The basal planes of the $2 \mathrm{D} \mathrm{WS}_{2}$ crystals display a low reactivity toward the $\mathrm{WF}_{6}$ precursor. $\mathrm{A}_{2}$ plasma exposure reduces the adsorbed $W_{\mathrm{x}}$ species from the initial $\mathrm{VI}+$ oxidation state, and enables subsequent $\mathrm{WF}_{6}$ adsorption by creating reactive surface sites. Afterwards, $\mathrm{WS}_{2}$ forms through oxidation reaction with $\mathrm{H}_{2} \mathrm{~S}$, yielding $\mathrm{W}$ in the final $+\mathrm{IV}$ oxidation state. Hence, the $\mathrm{H}_{2}$ plasma and $\mathrm{H}_{2} \mathrm{~S}$ reactions need to be carefully balanced to control the partial reduction of $\mathrm{W}$ atom from $+\mathrm{VI}$ to +IV oxidation in $\mathrm{WS}_{2}$.

The crystals created by 50-100 PEALD reaction cycles consist of $\mathrm{WS}_{2}$, as the S/W ratio approaches $2.1 \pm 0.2$ despite the air exposure (XPS, Figure 3a-b). This indicates that these $\mathrm{WS}_{2}$ crystals become more resistant to oxidation in air as their size increases because of a higher volume-to-surface ratio. In contrast, the $\mathrm{WS}_{2} / \mathrm{WO}_{\mathrm{x}}$ ratio is low and even decreases slightly during the initial stage of the PEALD process (Figure $3 \mathrm{a}, \mathrm{b}$ ). The $\mathrm{WS}_{2} / \mathrm{WO}_{\mathrm{x}}$ ratio after 25 PEALD reaction cycles is slightly lower as compared to the initial seed. This may suggest that the initial $\mathrm{H}_{2}$ plasma conditions in the PEALD process are too reducing for the small amount of available $\mathrm{WS}_{2}$, forming residual $\mathrm{W}$ that readily oxidizes to $\mathrm{WO}_{3}$ and $\mathrm{WO}_{2}$ during air exposure ${ }^{32}$. Reducing the $\mathrm{H}_{2}$ plasma reactivity during the initial PEALD process through plasma power and reactor pressure may provide improved control over the compositional ratio $\mathrm{S} / \mathrm{W}$.

Furthermore, the $\mathrm{WS}_{2}$ crystals are Raman active, confirming their 2D structure (Figure 3c-d). The full-width at half maximum (fwhm) of the prominent longitudinal acoustic Raman mode 2LA(M) at the M point of the Brillouin zone qualitatively relates to local disorder in the in-plane $\mathrm{WS}_{2}$ crystal lattice structure ${ }^{36}$. The $\mathrm{WS}_{2}$ seeds after selective conversion and anisotropic growth by PEALD display a $2 \mathrm{LA}$ fwhm in line with $\mathrm{WS}_{2}$ PEALD layers deposited on bare $\mathrm{SiO}_{2}$ starting surfaces of $(13.1 \pm 0.3) \mathrm{cm}^{-1}$, for similar amounts of deposited $\mathrm{WS}_{2}$. Hence, during the PEALD process, the $\mathrm{WS}_{2}$ seeds develop into $\mathrm{WS}_{2}$ crystals with similar crystalline quality as compared to $\mathrm{WS}_{2}$ PEALD layers deposited on bare $\mathrm{SiO}_{2}$ in earlier work ${ }^{33}$. However, the 2LA(M) peak remains broader when compared to $\mathrm{WS}_{2}$ natural crystal flakes [i.e., $(8.9 \pm 0.5) \mathrm{cm}^{-1}$ ]. Indeed, the $2 \mathrm{LA}(\mathrm{M})$ peak broadens when the deposited crystals possess inter- and intragrain defects (e.g., grain boundary, vacancy), and/or a random orientation in the (0001) plane as expected in absence of a template. The $\mathrm{WS}_{2}$ seeds do not exhibit a PL response, as expected for seeds that consist of five or more layers.

During $\mathrm{WS}_{2}$ PEALD, the $\mathrm{WS}_{2}$ seed dimensions increase anisotropic, as determined from the corresponding AFM height profile (Figure 4a,c). After 75 PEALD reaction cycles, the seeds can develop into $\mathrm{WS}_{2}$ crystals with lateral dimensions approaching $90 \mathrm{~nm}$, whereas the height increases with only a few $\mathrm{nm}$ as compared to the initial height of the seeds after selective conversion of $12.0 \pm 0.4 \mathrm{~nm}$. 


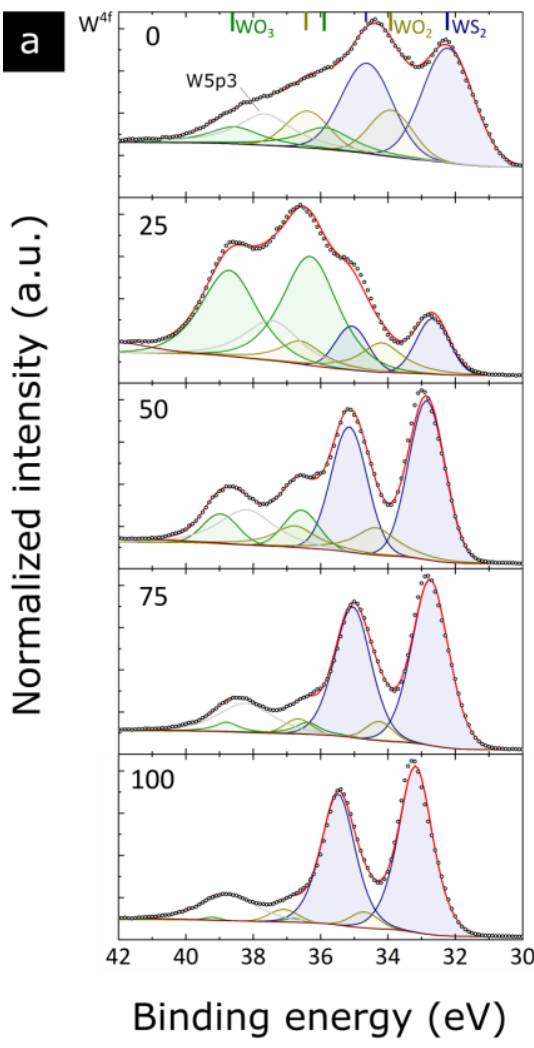

b

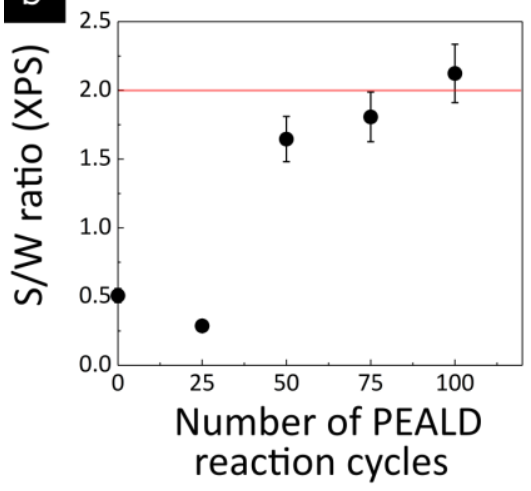

c

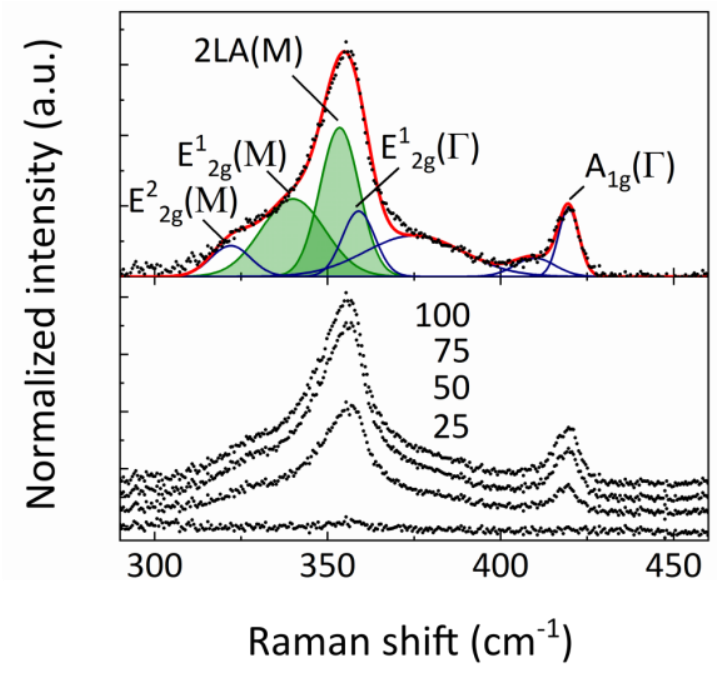

d

Absolute monolayer content (number of monolayers)

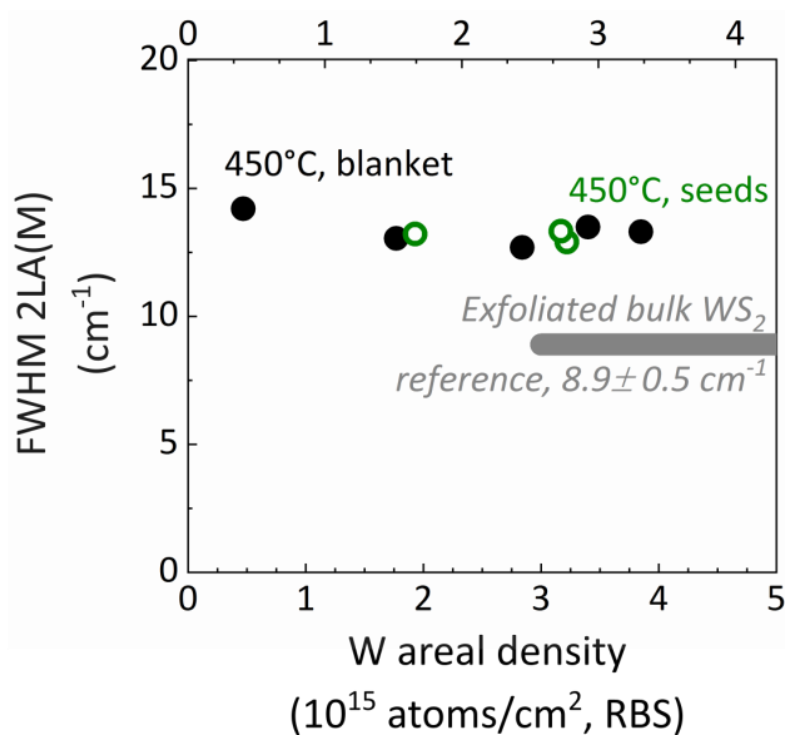

Figure 3. (a) Deconvoluted W4f XPS spectra of $\mathrm{WS}_{2}$ crystals after 0 , $25,50,75$ and 100 PEALD reaction cycles from $\mathrm{WS}_{2}$ seeds in array 2 , using doublet for $\mathrm{WS}_{2}$ (starting at $\sim 32.2 \mathrm{eV}$ ), $\mathrm{WO}_{2}$ (starting at $\sim 33.9 \mathrm{eV}$ ), and $\mathrm{WO}_{3}$ (starting at $\sim 35.9 \mathrm{eV}$ ). W5p3 spectral line and $\mathrm{WO}_{3}$ plasmon loss peak are included at $\sim 38 \mathrm{eV}$ and $\sim 42 \mathrm{eV}$. (b) The corresponding S/W ratio of the $\mathrm{WS}_{2}$ crystals as a function of the number of PEALD reaction cycles, as determined from calculated atomic concentrations of W4f and S2p surface spectra. (c) Deconvoluted Raman spectrum (top) and Raman signatures of $\mathrm{WS}_{2}$ crystals for different number of PEALD reaction cycles (bottom). Characteristic Raman peaks at $532 \mathrm{~nm}$ laser excitation wavelength, include second order longitudinal acoustic Raman mode 2LA(M), and first-order in-plane and out-of-plane optical modes $E^{1}{ }_{2 g}(\Gamma)$ and $A_{1 g}(\Gamma)$. (d) $2 \mathrm{LA}(\mathrm{M})$ fwhm of $\mathrm{WS}_{2}$ crystals after $\mathrm{WS}_{2}$ PEALD from $\mathrm{WS}_{2}$ seeds as a function of amount of deposited WS $\mathrm{W}_{2}$ (by RBS). The fwhm is compared to PEALD WS 2 and exfoliated $\mathrm{WS}_{2}$ flakes on blanket dielectric substrates.

Furthermore, the $\mathrm{WS}_{2}$ crystal basal planes attain an orientation parallel to the starting surface during the PEALD process, which suggests that such (0002) texture also exists in the $\mathrm{WS}_{2}$ seeds (Figure 2c,d). Indeed, the $\mathrm{WS}_{2}$ basal planes develop a preferential orientation along [0001] crystal direction, as evidenced by the relatively smooth topography and anisotropic shape of the AFM height profile (Figure 4c). As the lateral 
growth proceeds from the seeds, the seeds must therefore also consist of $\mathrm{WS}_{2}$ with a $2 \mathrm{D}$ structure and (0002) texture before air exposure, in agreement with our initial assumption. In contrast, larger $\mathrm{WS}_{2}$ patterns or $\mathrm{WS}_{2}$ thin films obtained by conversion of blanket Si precursor layers, develop a larger tilt misorientation of the (0002) $\mathrm{WS}_{2}$ crystal basal planes with a crystal grain size of $5-10 \mathrm{~nm}^{31}$. Hence, nanoscale Si structures convert differently into $\mathrm{WS}_{2}$ crystals with horizontal basal planes. Presumably, crystal growth is less hindered by neighbouring $\mathrm{WS}_{2}$ crystals or intermediate Si clusters. Note that the inplane orientation of $\mathrm{WS}_{2}$ crystals is not controlled in the current experiments due to absence of a template.

In view of (0002) texture of the $\mathrm{WS}_{2}$ seeds, adsorbed precursor species preferentially incorporate at the more reactive crystal edges. However, the $\mathrm{H}_{2}$ plasma can also create reactive surface sites for $\mathrm{WF}_{6}$ precursor adsorption on the $\mathrm{WS}_{2}$ basal plane, resulting in nucleation of a crystal in the next $\mathrm{WS}_{2}$ layer, in line with the anisotropic island-like growth mode proposed in earlier work ${ }^{33,35}$.

In contrast, for some $\mathrm{WS}_{2}$ seeds the surface roughens which increases the uncertainty on the extracted seed height, especially for larger number of PEALD reaction cycles (Figure $4 \mathrm{~b}, \mathrm{~d}$ ). A portion of the (0002) crystal planes can develop a tilt-misorientation with respect to the underlying starting surface (e.g., white dots in AFM images, Figure 4). That is, the seeds can possess mosaicity where the (0002) crystal planes orient out of the horizontal plane of the starting surface. Mosaicity in the $\mathrm{WS}_{2}$ PEALD has been observed in our earlier work and in literature $33,37,38$. This can be caused through disorder in the 2D structure of the starting seed (e.g., defects, grain boundaries), or the roughness of the starting surface. In addition, some $\mathrm{WS}_{2}$ crystals grow in the vertical dimension through dislocation-driven spiral growth where a slightly tilted (0002) crystal plane develops into $\mathrm{WS}_{2}$ spiral $^{39}$. Due to the increased density of exposed reactive edge sites, such spiralling crystals are self-sustaining and can lead to rapid increase in $\mathrm{WS}_{2}$ seed height.

Analysis of the evolution of $\mathrm{WS}_{2}$ crystal dimensions during ALD further confirms the anisotropic growth and moreover can reveal insight in the growth mechanism of controlled seeding. The lateral size increases fairly linearly during PEALD and the lateral GPC is independent of the considered seed size (Figure 2b). This lateral GPC is $0.30 \pm 0.03 \mathrm{~nm} /$ cycle, assuming a linear fit between 0 and 100 PEALD reaction cycles. In contrast, the vertical GPC is only $0.07 \pm 0.02 \mathrm{~nm} /$ cycle. Further, the lateral GPC from the $\mathrm{WS}_{2}$ seeds is lower than the lateral GPC for $\mathrm{WS}_{2}$ crystals on blanket dielectric substrates, which is $0.44 \pm 0.02 \mathrm{~nm} /$ cycle.

A constant GPC during controlled seeding by PEALD should not necessarily be expected, as a diffusion-mediated anisotropic island growth mechanism has been proposed for $\mathrm{WS}_{2}$ PEALD on dielectric substrates in earlier work ${ }^{33}$. Adsorbed precursor species diffuse until they reach the edges of $\mathrm{WS}_{2}$ seed, where they are incorporated in the crystal. The preferential lateral growth from the edges of the $\mathrm{WS}_{2}$ seeds can be understood from the anisotropic reactivity of $\mathrm{WS}_{2}$ crystals: the basal plane is self-passivating and therefore less reactive as compared to the edges of the $\mathrm{WS}_{2}$ crystals. Undesired $\mathrm{WS}_{2}$ nucleation by agglomeration on the $\mathrm{Si}_{3} \mathrm{~N}_{4}$ surface also occurs (AFM). The lateral GPC of the crystals will depend on the rates of precursor adsorption, diffusion and agglomeration and can moreover depend on the distance between the $\mathrm{WS}_{2}$ seeds ${ }^{40,41}$. The processes contributing to film growth during the initial nucleation regime of ALD, i.e. adsorption, surface diffusion and reaction, may be different than those during bulk growth. Saturation of the surface reactions is therefore not guaranteed, and the GPC increase may differ from linearity. As such, the fairly linear evolution during the first 100 PEALD reaction cycles might be specific for the considered $\mathrm{WS}_{2}$ seed array geometry.

One can expect the lateral size of the $\mathrm{WS}_{2}$ crystals to increase further by additional PEALD reaction cycles. However, the atomic force micrographs after 100 and 150 PEALD reaction cycles show defects appearing in between the $\mathrm{WS}_{2}$ crystals (Figure $2 \mathrm{a}, 4 \mathrm{a}, \mathrm{b}$ ). This may point toward unintentional $\mathrm{WS}_{2}$ deposition in the area between the growing crystals. Indeed, selectivity during ALD is often found to decrease after extensive ALD reaction cycles. An extension to large crystal size would require high selectivity and the development of defect mitigation strategies ${ }^{42}$. The selectivity could be 
improved by dielectric surface functionalization, by decreasing adsorption rate and/or enhancing diffusion rate, or by using other ALD processes.

Overall, the controlled seeding concept can in principle allow the formation of monocrystalline $\mathrm{WS}_{2}$ crystals provided that the $\mathrm{WS}_{2}$ seed is monocrystalline. The nanocrystalline nature of the seed in the current experiment at least partly disturbs the final $\mathrm{WS}_{2}$ structures, which are also nanocrystalline. Developing monocrystalline seeds could be possible by either reducing the dimensions of the Si precursor patterns, or by considering other conversion chemistries.

\section{a}
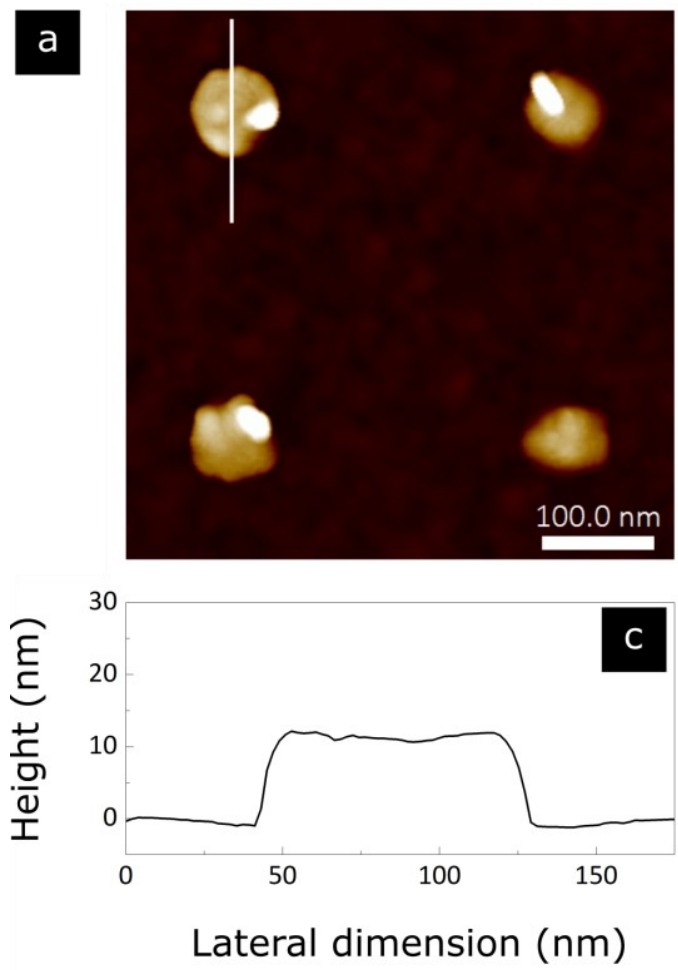

b

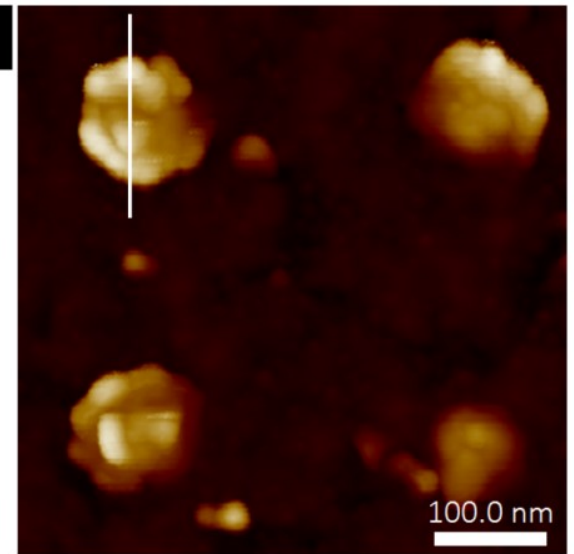

$30 \mathrm{~nm}$

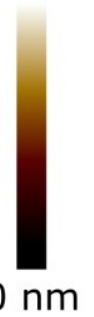

$0 \mathrm{~nm}$

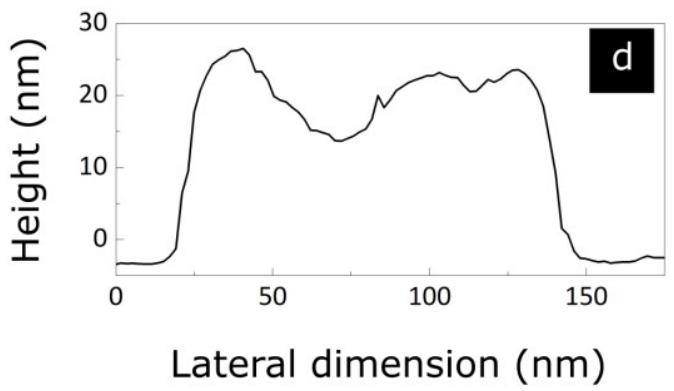

Figure 4: (a-b) Atomic force micrographs $(500 \mathrm{~nm}$ x $500 \mathrm{~nm})$ of the $\mathrm{WS}_{2}$ seeds after 75 and 150 PEALD reaction cycles. White dotted line corresponds to the AFM height profiles shown in (c) after 75 and (d) 150 PEALD reaction cycles.

\section{CONCLUSION}

Anisotropic growth of 2D materials during PEALD has been applied in controlled seeding of 2D crystals. $\mathrm{WS}_{2}$ crystals were grown by PEALD from $2 \mathrm{D}$ material seeds at predetermined locations on a large area substrate. Their lateral crystal size can be tuned by the number of PEALD reaction cycles.

In principle, this approach can be extended to other 2D materials, and heterostructures thereof. Better understanding of the growth and reaction mechanisms for anisotropic growth during ALD can contribute to control the material shape and structure. Further research can focus on improving the structural quality of the nanoscale seed as it greatly affects the final structures. In addition, the selectivity can be increased by process optimization, surface functionalization as well as better understanding of the impact of the pattern geometry. 


\section{Supplementary Material}

Supplemental material is available online and further details the proposed concept of controlled seeding and provides supplemental atomic force micrographs of the Si nanodots after immersion lithography and etch development, and of the $\mathrm{WS}_{2}$ seeds after area-selective conversion.

\section{Acknowledgements}

This project has received funding from the European Union's Horizon 2020 research and innovation program under the Marie Sklodowska-Curie grant agreement No 704004. Also, B.G. acknowledges the financial support from Flanders Innovation \& Entrepreneurship (VLAIO) under project no. 141640, and the imec Industrial Affiliation Beyond CMOS program. We are thankful for the support from Thierry Conard for the XPS measurements, Johan Desmet and Johan Meersschaut for the RBS measurements, Danielle Vanhaeren for the AFM measurements, and Thomas Nuytten for the Raman measurements.

The data that support the findings of this study are available from the corresponding author upon reasonable request.

\section{Conflicts of interest}

There are no conflicts to declare.

\section{Notes and references}

‡ Supporting information (SI) is provided free of charge.

1. Manzeli, S., Ovchinnikov, D., Pasquier, D., Yazyev, O. V. \& Kis, A. 2D transition metal dichalcogenides. Nature Reviews Materials 2, 17033 (2017).

2. Chhowalla, M. et al. The chemistry of two-dimensional layered transition metal dichalcogenide nanosheets. Nat Chem 5, 263-275 (2013).

3. Wang, Q. H., Kalantar-Zadeh, K., Kis, A., Coleman, J. N. \& Strano, M. S. Electronics and optoelectronics of two-dimensional transition metal dichalcogenides. Nat. Nanotechnol. 7, 699-712 (2012).

4. Cai, Z., Liu, B., Zou, X. \& Cheng, H.-M. Chemical Vapor Deposition Growth and Applications of Two-Dimensional Materials and Their Heterostructures. Chem. Rev. 118, 6091-6133 (2018).

5. Ly, T. H. et al. Misorientation-angle-dependent electrical transport across molybdenum disulfide grain boundaries. Nat. Commun. 7, 10426, pp 1-7 (2016).

6. Zhang, X. et al. Diffusion-Controlled Epitaxy of Large Area Coalesced $\mathrm{WSe}_{2}$ Monolayers on Sapphire. Nano Lett. 18, 1049-1056 (2018).

7. Brems, S. et al. (Invited) Graphene Synthesis and Transfer Improvements for Applications in the Semiconductor Industry. ECS Trans. 77, 3-13 (2017).

8. Kang, K. et al. High-mobility three-atom-thick semiconducting films with wafer-scale homogeneity. Nature 520, 656-660 (2015).

9. Grillo, F., La Zara, D., Mulder, P., Kreutzer, M. T. \& Ruud van Ommen, J. Oriented Attachment and Nanorod Formation in Atomic Layer Deposition of $\mathrm{TiO}_{2}$ on Graphene Nanoplatelets. J. Phys. Chem. C 122, 19981-19991 (2018).

10. Kim, C. et al. Copper Nanowires with a Five-Twinned Structure Grown by Chemical Vapor Deposition. Adv. Mater. 20, 1859-1863 (2008).

11. Zhang, W., Dey, G., Mandia, D. J. \& Barry, S. T. Using a Vapor-Phase Surfactant to Control Gold Metal Plate Growth. Advanced Materials Interfaces 4, 1600864 (2017).

12. Merckling, C. et al. Heteroepitaxy of InP on Si(001) by selective-area metal organic vaporphase epitaxy in sub-50 nm width trenches: The role of the nucleation layer and the recess engineering. J. Appl. Phys. 115, 023710, pp 1-6 (2014).

13. Jiang, S. et al. Evolution of (001) and (111) facets for selective epitaxial growth inside submicron trenches. Journal of Applied Physics 115, 023517 (2014). 
14. Leonhardt, A. et al. Improving MOCVD $\mathrm{MoS}_{2}$ Electrical Performance: Impact of Minimized Water and Air Exposure Conditions. IEEE Electron Device Lett. 38, 1606-1609 (2017).

15. Han, G. H. et al. Seeded growth of highly crystalline molybdenum disulphide monolayers at controlled locations. Nat. Commun. 6, 6128, pp 1-6 (2015).

16. Su, G. et al. Chemical Vapor Deposition of Thin Crystals of Layered Semiconductor $\mathrm{SnS}_{2}$ for Fast Photodetection Application. Nano Lett. 15, 506-513 (2015).

17. Sun, D. et al. Chemical vapor deposition growth of a periodic array of single-layer $\mathrm{MoS}_{2}$ islands via lithographic patterning of an $\mathrm{SiO}_{2}$ /Si substrate. 2D Mater. 2, 045014, pp 1-5 (2015).

18. Wang, Z. et al. Metal Induced Growth of Transition Metal Dichalcogenides at Controlled Locations. Sci. Rep. 6, 38394, pp 1-7 (2016).

19. Godin, K., Kang, K., Fu, S. \& Yang, E.-H. Increased monolayer domain size and patterned growth of tungsten disulfide through controlling surface energy of substrates. J. Phys. D: Appl. Phys. 49, 325304, pp 1-10 (2016).

20. Wang, X., Kang, K., Chen, S., Du, R. \& Yang, E.-H. Location-specific growth and transfer of arrayed $\mathrm{MoS}_{2}$ monolayers with controllable size. 2D Mater. 4, 025093, pp 1-7 (2017).

21. Bersch, B. M. et al. Selective-area growth and controlled substrate coupling of transition metal dichalcogenides. 2D Mater. 4, 025083, pp 1-10 (2017).

22. Cheng, C. et al. First demonstration of 40-nm channel length top-gate $\mathrm{WS}_{2} \mathrm{pFET}$ using channel area-selective CVD growth directly on $\mathrm{SiO}_{x} / \mathrm{Si}$ substrate. in 2019 Symposium on VLSI Technology T244-T245 (2019). doi:10.23919/VLSIT.2019.8776498.

23. Leskelä, M. \& Ritala, M. Atomic Layer Deposition Chemistry: Recent Developments and Future Challenges. Angew. Chem., Int. Ed. 42, 5548-5554 (2003).

24. Leskelä, M., Mattinen, M. \& Ritala, M. Review Article: Atomic layer deposition of optoelectronic materials. Journal of Vacuum Science \& Technology B 37, 030801 (2019).

25. Hao, W., Marichy, C. \& Journet, C. Atomic layer deposition of stable 2D materials. 2D Mater. 6, 012001 (2018).

26. Pulinthanathu Sree, S. et al. Anisotropic Atomic Layer Deposition Profiles of $\mathrm{TiO}_{2}$ in Hierarchical Silica Material with Multiple Porosity. Chem. Mater. 24, 2775-2780 (2012).

27. Shi, J. et al. Electron Microscopy Observation of $\mathrm{TiO}_{2}$ Nanocrystal Evolution in HighTemperature Atomic Layer Deposition. Nano Lett. 13, 5727-5734 (2013).

28. Delabie, A. et al. Low temperature deposition of $2 \mathrm{D} W S 2$ layers from $\mathrm{WF}_{6}$ and $\mathrm{H}_{2} \mathrm{~S}$ precursors: impact of reducing agents. Chem. Commun. 51, 15692-15695 (2015).

29. Heyne, M. H. et al. Two-dimensional WS 2 nanoribbon deposition by conversion of prepatterned amorphous silicon. Nanotechnology 28, 04LT01, pp 1-5 (2017).

30. Yu, M. L., Ahn, K. Y. \& Joshi, R. V. Surface reactions in the chemical vapor deposition of tungsten using $\mathrm{WF}_{6}$ and $\mathrm{SiH}_{4}$ on Al, PtSi, and TiN. J. Appl. Phys. 67, 1055-1061 (1990).

31. Heyne, M. H. et al. The conversion mechanism of amorphous silicon to stoichiometric $\mathrm{WS}_{2}$.J. Mater. Chem. C 6, 4122-4130 (2018).

32. Groven, B. et al. Plasma-Enhanced Atomic Layer Deposition of Two-Dimensional $\mathrm{WS}_{2}$ from $\mathrm{WF}_{6}, \mathrm{H}_{2}$ Plasma, and $\mathrm{H}_{2} \mathrm{~S}$. Chem. Mater. 29, 2927-2938 (2017).

33. Groven, B. et al. Two-Dimensional Crystal Grain Size Tuning in $\mathrm{WS}_{2}$ Atomic Layer Deposition: An Insight in the Nucleation Mechanism. Chem. Mater. 30, 7648-7663 (2018).

34. Meersschaut, J. \& Vandervorst, W. High-throughput ion beam analysis at imec. Nucl. Instrum. Methods Phys. Res., Sect. B 406, 25-29 (2017).

35. Groven, B. et al. Nucleation mechanism during $\mathrm{WS}_{2}$ plasma enhanced atomic layer deposition on amorphous Al203 and sapphire substrates. J. Vac. Sci. Technol., A 36, 01A105, pp 1-11 (2017).

36. Berkdemir, A. et al. Identification of individual and few layers of $\mathrm{WS}_{2}$ using Raman Spectroscopy. Sci. Rep. 3, 1755, pp 1-8 (2013).

37. Balasubramanyam, S. et al. Edge-Site Nanoengineering of $\mathrm{WS}_{2}$ by Low-Temperature PlasmaEnhanced Atomic Layer Deposition for Electrocatalytic Hydrogen Evolution. Chem. Mater. 31, 5104-5115 (2019).

38. Balasubramanyam, S. et al. Probing the Origin and Suppression of Vertically Oriented Nanostructures of 2D WS 2 Layers. ACS Appl. Mater. Interfaces 12, 3873-3885 (2020). 
39. Sarma, P. V., Patil, P. D., Barman, P. K., Kini, R. N. \& Shaijumon, M. M. Controllable growth of few-layer spiral $\mathrm{WS}_{2}$. RSC Adv. 6, 376-382 (2015).

40. Carlsson, J.-0. Selective vapor-phase deposition on patterned substrates. Critical Reviews in Solid State and Materials Sciences 16, 161-212 (1990).

41. Richey, N. E., de Paula, C. \& Bent, S. F. Understanding chemical and physical mechanisms in atomic layer deposition. J. Chem. Phys. 152, 040902 (2020).

42. Mackus, A. J. M., Merkx, M. J. M. \& Kessels, W. M. M. From the Bottom-Up: Toward AreaSelective Atomic Layer Deposition with High Selectivity. Chem. Mater. 31, 2-12 (2019).

Electronic Supplementary Information (ESI) available: experimental details. See DOI: 10.1039/x0xx00000x 